\title{
Electrically tunable ring resonators incorporating nematic liquid crystals as cladding layers
}

\author{
Brett Maune ${ }^{\text {a) }}$ \\ Department of Applied Physics, California Institute of Technology, Pasadena, California 91125 \\ Rhys Lawson \\ Department of Chemistry, University of Washington, Seattle, Washington 98195
}

Cary Gunn

Luxtera, Carlsbad, California 92008

Axel Scherer

Department of Applied Physics, California Institute of Technology, Pasadena, California 91125

Larry Dalton

Department of Chemistry, University of Washington, Seattle, Washington 98195

(Received 20 August 2003; accepted 2 October 2003)

\begin{abstract}
We have demonstrated electrical tuning in ring resonators fabricated from silicon-on-insulator wafers by incorporating nematic liquid crystals (NLCs) as the waveguide top and side cladding material. Photolithographically defined electrodes aligned around the ring resonator were used to control the orientation of the NLCs to modulate the cladding refractive index and, hence, the resonant wavelengths of the ring resonator. (C) 2003 American Institute of Physics.
\end{abstract}

[DOI: $10.1063 / 1.1630370]$

Microring resonators, fabricated with conventional semiconductor processing methods in silicon, offer significant advantages over the existing telecommunication filter technology and may be the foundation of future dense-wavelengthdivision-multiplexing (DWDM) filters. $^{1-5}$ The high refractive index (RI) contrast available in silicon-oninsulator (SOI) ring resonators enables low loss and high- $Q$ filters fabricated with radii down to a few microns. ${ }^{6,7}$ Such resonators can be designed as notch filters for adding or dropping individual channels in the telecommunication bands and can be densely integrated in photonic networks. For reconfigurable DWDM systems, and to compensate for temperature changes, it is desirable to tune the precise channel frequency dropped by such resonator add/drop multiplexers.

Two primary methods exist to control the optical path length of a ring resonator and thus tune its resonant frequency. To statically tune a ring resonator one can either adjust the physical dimensions (in particular its circumference) or the refractive indices of the constituent materials of the resonator. Dynamically tunable resonators provide another level of functionality over statically tuned resonators and are most practically obtained by controlling the refractive indices of the constituent materials. Dynamic tuning is commonly achieved by thermally changing the RI, traditionally by introducing a heater close to the resonator. ${ }^{8}$ However, power dissipation may provide a serious problem in such tunable ring resonator designs, especially when many resonators have to be integrated in a DWDM multiplexing system. In this letter we demonstrate the dynamic tuning of a ring resonator by changing the RI of its cladding via the

\footnotetext{
a) Author to whom correspondence should be addressed; electronic mail: bmaune@caltech.edu
}

orientation of the nematic liquid crystals (NLC).

The resonator system under study, as shown in Fig. 1, was fabricated from a SOI wafer with silicon thickness of $205 \mathrm{~nm}$ and oxide thickness of $1 \mu \mathrm{m}$, ring radius of $5 \mu \mathrm{m}$, and ring and waveguide widths of $500 \mathrm{~nm}$. The resonator was coupled to one waveguide, which served as both the input and output port and was separated from the resonator by a $100 \mathrm{~nm}$ gap. Modulation electrodes were then photolithographically defined and deposited using standard lift-off processing. The left and right electrodes were approximately $4.0 \mu \mathrm{m}$ wide and were spaced about 400 and $300 \mathrm{~nm}$ from the resonator, respectively (Fig. 1). The modulation electrodes were designed to preferentially orient the directors of the NLC molecules parallel (azimuthally oriented) to the resonator. To minimize their electrostatic energy, the NLC molecules rotate and orient their directors along the direction

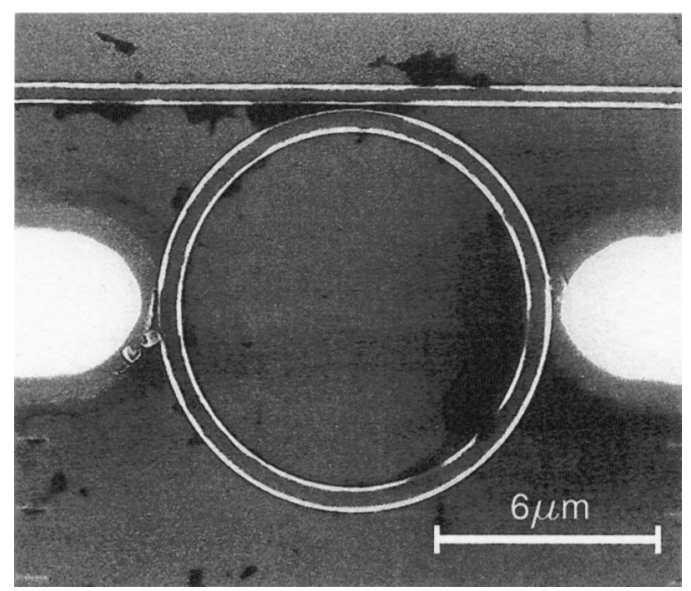

FIG. 1. A scanning electron microscopy image of the ring resonator with modulation electrodes (and NLC remnants). 


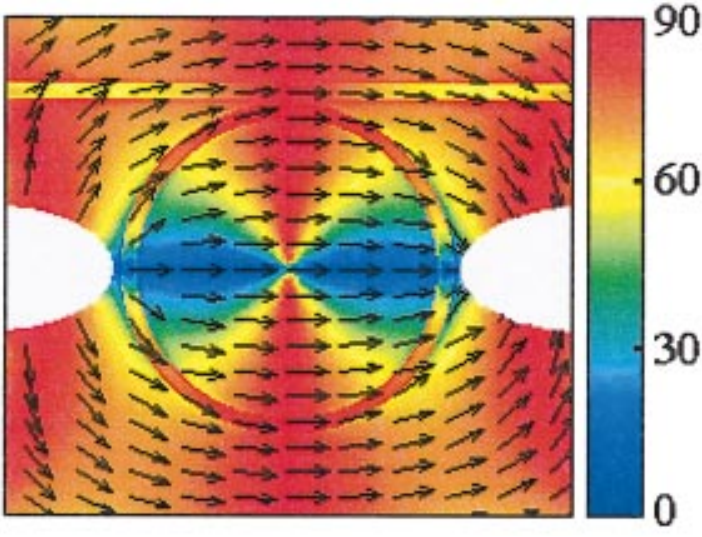

FIG. 2. (Color) Electric field generated by electrodes. Arrows indicate direction of field and color-scale indicates angular difference between presumed director alignment (parallel to electric field direction) and radial axis.

of the applied electric field. ${ }^{9}$ Figure 2 shows the simulated electric field generated by the electrodes designed for the azimuthally biased orientation of the NLC. The NLC E63 $\left(n_{e}=1.744, n_{o}=1.517\right.$ at $\left.589 \mathrm{~nm}\right)$ from Merck was used for the resonator top and side cladding material and was applied to the resonator by spinning at $4000 \mathrm{rpm}$ for $40 \mathrm{~s}$. Light from a tunable semiconductor laser was grating coupled, both into and out of the respective ports. The grating couplers selectively coupled and transmitted the waveguide's single TE mode (electric field in the plane of the resonator). The resonator's resonances were measured by recording the power from the output port while sweeping the wavelength of the laser. Scans were taken with different electric potentials applied across the electrodes.

The resonances of the rings correspond to the wavelengths of light coupling into the resonator which undergo a phase shift of an integer multiple of $2 \pi$ upon one round trip in the resonator. The resonance wavelengths can therefore be determined by modifying the Fabry-Pérot etalon resonance equation to obtain ${ }^{10}$

$$
\lambda_{m}=2 \pi R n_{\mathrm{eff}} / m,
$$

where $m=1,2,3 \ldots ; \lambda_{m}=$ wavelength of the $m$ th resonator mode; $R$ =radius of resonator (measured from the center to midpoint of ring); and $n_{\text {eff }}=$ effective RI of waveguide mode.

From Eq. (1) we can infer that the essence of tuning the resonator lay in changing $n_{\text {eff }}$. Fortunately, the TE waveguide mode contains evanescent tails that penetrate into the NLC cladding and consequently $n_{\text {eff }}$ is partly dependent upon the NLC cladding RI, $n_{\text {clad }}$.

For the TE guided mode, the contribution to $n_{\text {clad }}$ of a NLC molecule is given by ${ }^{11}$

$$
1 / n_{\text {clad }}^{2}=\cos ^{2}(\theta) / n_{e}^{2}+\sin ^{2}(\theta) / n_{o}^{2}
$$

where $\theta=$ the angle between the NLC director and the radial axis.

With the electrode configuration shown in Fig. 2, the application of an electric field will preferentially orient the NLC azimuthally. Equation (2) shows that $n_{\text {clad }}$ is expected to decrease towards $n_{o}$, in turn decreasing $n_{\text {eff }}$. A decreasing $n_{\text {eff }}$ causes the resonances to shift to shorter wavelengths, Eq. (1). We recorded wavelength scans while applying potentials of $0-20 \mathrm{~V}$ across the electrodes and monitored the resonator Downloaded 17 Feb 2006 to 131.215.225.176. Redistribution subje

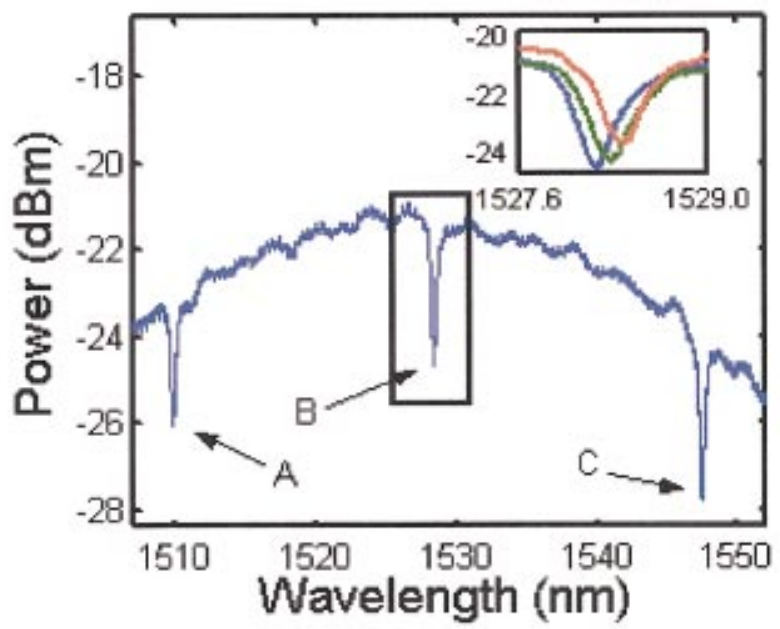

(a)

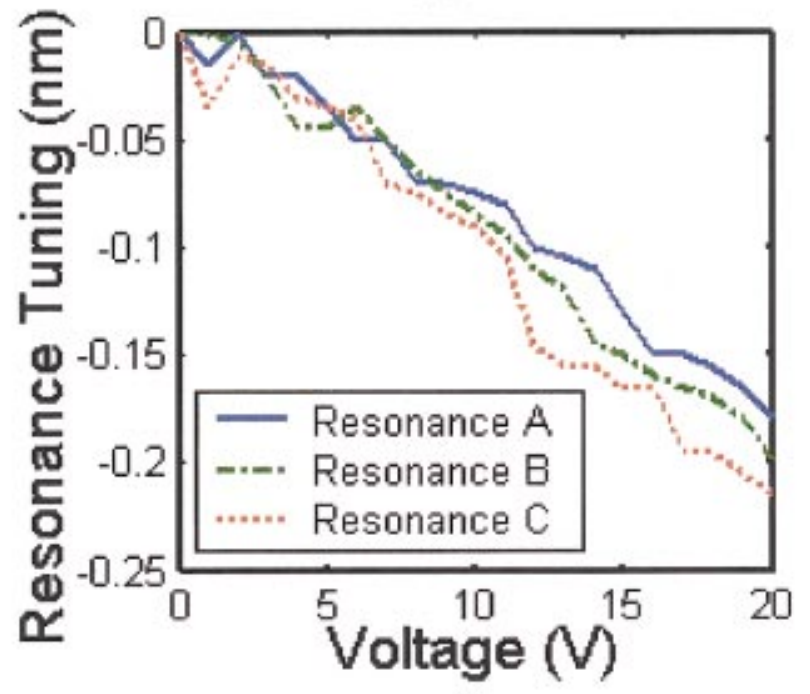

(b)

FIG. 3. (Color) (a) Wavelength scan showing three resonances A, B, and C at $0 \mathrm{~V}$. (inset) Close-up of resonance $\mathrm{B}$ at 0,10 , and $20 \mathrm{~V}$. (b) The wavelength tuning of each resonance vs applied voltage.

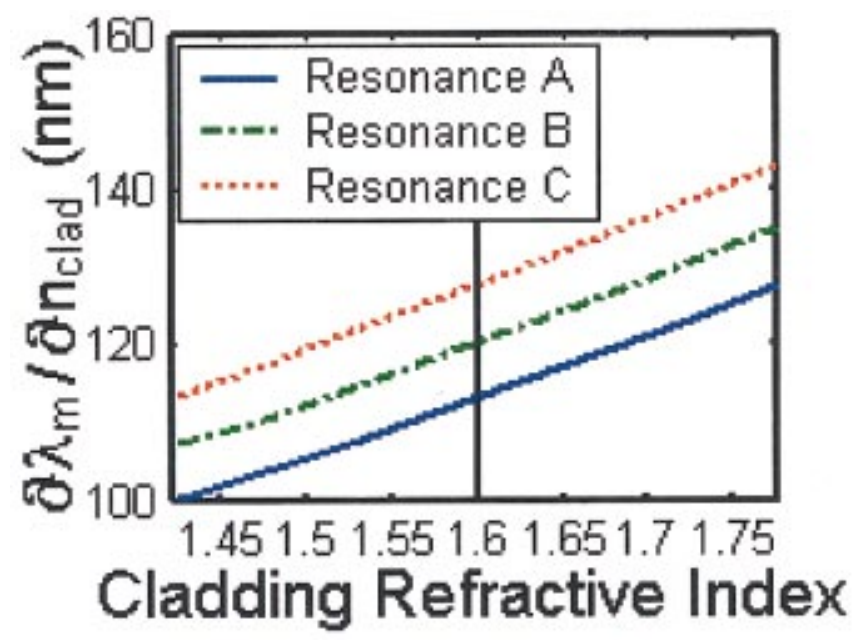

FIG. 4. (Color) Plot of $\partial \lambda_{m} / \partial n_{\text {clad }}$ vs $n_{\text {clad }}$ showing resonance wavelength sensitivity to $n_{\text {clad }}$ changes. Vertical line indicates $n_{\text {clad }}$ corresponding to random NLC orientation.

to AIP license or copyright, see http://apl.aip.org/apl/copyright.jsp 
drop frequency with a tunable laser. The resulting data for three resonances are shown in Fig. 3. Note that the resonance shift is in the opposite direction as expected if the resonator were being heated (by absorption of the laser or leakage currents across the electrodes). ${ }^{12}$

We obtained a maximum resonance shift of approximately $0.22 \mathrm{~nm}(27.5 \mathrm{GHz}$ at $1.55 \mu \mathrm{m})$ by applying $20 \mathrm{~V}$. To correlate our experimental results with the degree of NLC alignment, we start with a modified form of Eq. (1) and obtain

$$
\Delta \lambda_{m}=\left(\partial n_{\text {eff }} / \partial n_{\text {clad }}\right) \lambda_{m} \Delta n_{\text {clad }} / n_{\text {eff }},
$$

where $\Delta \lambda_{m}=$ the shift in resonance wavelength; $\partial n_{\text {eff }} / \partial n_{\text {clad }}=$ the derivative of $n_{\text {eff }}$ with respect to $n_{\text {clad }}$; and $\Delta n_{\text {clad }}=$ the change in the cladding RI due to NLC alignment.

We assumed that with zero applied electric field the NLC was randomly oriented. For E63, this corresponds to an $n_{\text {clad }}$ of 1.596. We subsequently used this $n_{\text {clad }}$ to simulate $n_{\text {eff }}$ and $\partial n_{\text {eff }} / \partial n_{\text {clad }}$ for the three resonant wavelengths under study. Then, by using Eq. (3) we solved for $\partial \lambda_{m} / \partial n_{\text {clad }}$ and obtained the resonance wavelength sensitivity to $n_{\text {clad }}$ (Fig. 4). Using this in conjunction with the wavelength shift data, we obtained a $\Delta n_{\text {clad }}$ of only -0.0016 for all three resonances. Based on our electrostatic modeling for the azimuthally biased electrode configuration, in the NLC saturation field regime we would expect a $\Delta n_{\text {clad }}$ of -0.0342 . Though the applied field was expected to exceed E63's saturation field of $0.52 \mathrm{~V} / \mu \mathrm{m}$, the resonances instead continued to shift through the maximum applied field. This indicated the applied electric field was insufficient to achieve complete NLC alignment and it partly explains the disparity between the calculated and measured $\Delta n_{\text {clad }}$ values. Another possible explanation for the disparity between $\Delta n_{\text {clad }}$ values is that the fabricated electrodes were not identical to the electrodes used in the electrostatics modeling. As can be seen in Fig. 2, the tuning electrodes used in this study align the NLCs azimuthally, radially, or in a linear combination of the two, depending on their position around the ring. Therefore, the actual resonance tuning results from the relative imbalance between azimuthal and radial NLC alignment, which in turn is extremely sensitive to the electrode geometry. The electric field generated by the fabricated electrodes may then differ substantially from the simulated field. This combined with NLC surface anchoring effects and a likely decreased birefringence at $1.55 \mu \mathrm{m}^{13}$ can explain the significant difference between measured and simulated $\Delta n_{\text {clad }}$ values.

As expected, the resonance shifts were independent of field polarity and were completely reversible. After removing the electric field, we observed the resonances return to their original wavelengths.

In conclusion, we have demonstrated an electrically tunable ring resonator using NLC as the cladding. The resonance wavelengths could be reversibly controlled up to a $0.22 \mathrm{~nm}$ range by applying an electric field across the resonator to align the NLC cladding. In the future we intend to investigate increasing the tuning range of the resonator and shortening the resettling time by using alternate electrode designs, higher electric fields, and a NLC alignment layer on the surface of the ring.

The authors would like to thank Luxtera, Inc., and especially Alex Dickinson for technical assistance with this research. Funding for this work by Jag Shah from DARPA under the CS-WDM program (Contract No. N00421-02-D3223) is also gratefully acknowledged.

${ }^{1}$ M. K. Chin, C. Youtsey, W. Zhao, T. Pierson, Z. Ren, S. L. Wu, L. Wang, Y. G. Zhao, and S. T. Ho, IEEE Photonics Technol. Lett. 11, 1620 (1999).

${ }^{2}$ B. E. Little, S. T. Chu, H. A. Haus, J. Foresi, and J.-P. Laine, J. Lightwave Technol. 15, 998 (1997).

${ }^{3}$ S. Suzuki, Y. Hatakeyama, Y. Kokubun, and S. T. Chu, J. Lightwave Technol. 20, 745 (2002).

${ }^{4}$ K. Oda, N. Takato, and H. Toba, J. Lightwave Technol. 9, 728 (1991).

${ }^{5}$ S. T. Chu, B. E. Little, W. Pan, T. Kaneko, S. Sato, and Y. Kokubun, IEEE Photonics Technol. Lett. 11, 691 (1999).

${ }^{6}$ D. Rafizadeh, J. P. Hagness, A. Taflove, K. A. Stair, and S. T. Ho, Opt. Lett. 22, 1244 (1997).

${ }^{7}$ D. Rafizadeh, J. P. Zhang, R. C. Tiberio, and S. T. Ho, J. Lightwave Technol. 16, 1308 (1998).

${ }^{8}$ P. Heimala, P. Katila, J. Aarnio, and A. Heinamaki, J. Lightwave Technol. 14, 2260 (1996).

${ }^{9}$ P. J. Collings, Liquid Crystals: Nature's Delicate Phase of Matter, 2 nd ed. (Princeton University Press, Princeton, 2002), Chap. 3, pp. 33, 34.

${ }^{10}$ B. E. A. Saleh and M. C. Teich, Fundamentals of Photonics, 1st ed. (Wiley, New York, 1991), Chap. 9, p. 314.

${ }^{11}$ Handbook of Liquid Crystals, 1st ed. (Wiley-VCH, New York, 1998), Vol. 1, pp. 215-217.

${ }^{12}$ We observed the resonance wavelengths shift in the opposite direction to longer wavelengths when increasing the temperature of the resonator with zero applied electric field.

${ }^{13}$ S.-T. Wu, C.-S. Hsu, and K.-F. Shyu, Appl. Phys. Lett. 74, 344 (1999). 